\title{
Update on therapies for acute ischemic stroke
}

\author{
Dean D. Kindler, M.D., M.A, GeOrge A. Lopez, M.D., Ph.D., \\ BRADFORD B. WORRALl, M.D., AND KaREN C. JOHNSTON, M.D., M.Sc. \\ Department of Neurology, University of Virginia, Charlottesville, Virginia
}

\begin{abstract}
Acute ischemic stroke is now considered a neurological emergency for which there are new therapies. Neurosurgeons and neurologists need to remain apprised of advances in this field. The authors discuss approved and emerging therapies for patients suffering from acute ischemic stroke, based on a review of recent publications. Currently, intravenous tissue-type plasminogen activator is the only Food and Drug Administration-approved therapy for acute ischemic stroke. Intraarterial delivery of thrombolytics is a promising treatment and may be effective in selected patients. Other therapies for acute cerebral ischemia are intriguing but still in the investigational stages.
\end{abstract}

KEY WORDS - acute ischemic stroke - intravenous thrombolytics • intraarterial thrombolytics • emerging therapy

With the incidence of new stroke approaching 700,000 per year in the US, ${ }^{7}$ neurologists and neurosurgeons will inevitably see and treat more patients with signs and symptoms of acute cerebral ischemia. The landscape of acute ischemic stroke therapies has been rapidly evolving during the last several years. Today there is an approved therapy for acute ischemic stroke, and other promising therapies are on the horizon. Practicing neurologists and neurosurgeons who treat stroke must remain in touch with the current standard of care in the acute setting.

\section{ESTABLISHED SYSTEMIC THERAPIES}

\section{Tissue-Type Plasminogen Activator}

In 1996 the FDA approved the administration of intravenous tPA for the treatment of ischemic stroke in patients presenting within 3 hours of symptom onset and meeting specified criteria (see Appendix). Approval was based on efficacy data obtained in the NINDS sponsored trial ${ }^{48}$ and subgroup analysis from the European trial. ${ }^{53}$ The NINDS trial was composed of two prospective, double-blind, placebo-controlled studies, allowing for the highest quality of evidence-based clinical study. Six hundred twenty-four

Abbreviations used in this paper: $\mathrm{CEA}=$ carotid endarterectomy; $\mathrm{CT}=$ computerized tomography; FDA = Food and Drug Administration; ICH = intracerebral hemorrhage; NINDS = National Institute for Neurological Disorders and Stroke; PROACT = Prolyse in Acute Cerebral Thromboembolism; r-proUK = recombinant prourokinase; $\mathrm{tPA}=$ tissue-type plasminogen activator; US = United States. patients were randomized to receive either intravenous tPA or placebo. The results of the trial demonstrated a benefit at 3 months for patients receiving intravenous tPA compared with placebo. Patients treated with intravenous tPA had at least a relative $30 \%$ greater likelihood of having minimal or no deficit at 3 months when compared with control patients. Although there was a significantly increased rate of symptomatic ICH in intravenous tPAtreated patients $(6.4 \%)$ compared with control patients $(0.6 \%)$, this was not associated with an increase in the number of deaths.

Post-hoc analysis of the NINDS data for patient pretreatment characteristics found no subgroup of patients who did not respond favorably to intravenous tPA therapy when compared with control patients, including such factors as age, stroke severity, and comorbid conditions such as diabetes. ${ }^{46}$ Separate data support the fact that patients aged 80 years and older who received intravenous tPA responded favorably, provided that clinicians adhered to the selection criteria as defined in the NINDS trial. ${ }^{56}$ Patients with severe strokes or early evidence of edema or mass effect as demonstrated by head CT scanning have higher rates of ICH in both control and tPA groups than patients with milder strokes. Nevertheless, each patient group examined benefited from intravenous tPA therapy if administrated within the 3 -hour window. ${ }^{47}$ Furthermore, patients with vertebrobasilar ischemia treated within 3 hours also demonstrate improvement with intravenous tPA. ${ }^{28}$ Further post-hoc analysis of the NINDS data suggests that patients receiving intravenous tPA in the earliest portion of the 3-hour window of opportunity were the most likely to have a good outcome. ${ }^{42}$ 
D. D. Kindler, et al.

Follow-up data obtained from the NINDS patients at 6 and 12 months show an effect of intravenous tPA of approximately the same magnitude of benefit as found at 3 months in the original NINDS trial. ${ }^{39}$ Open-label studies of intravenous tPA in both university and community hospital settings demonstrated good feasibility, safety, and efficacy when the NINDS protocol was followed. ${ }^{11}$ The general community hospital experience since FDA approval of tPA has also been encouraging. The published experience of community hospitals in which intravenous tPA is used demonstrate similar response and safety profiles..$^{25,29,30,55,59}$ In one study, however, a higher rate of ICH in intravenous tPA-treated patients was found than that published in the NINDS trial. ${ }^{37}$ The excess rate of $\mathrm{ICH}$ may have been influenced by protocol violations. Postmarketing surveillance data released this year support the use of the NINDS protocol by trained physicians at a wide variety of US medical centers. ${ }^{1}$

Attempts to extend the therapeutic window for intravenous tPA beyond 3 hours have been disappointing with both US and European data demonstrating no benefit and an excess occurrence of ICH compared with controls. ${ }^{12,23,31}$ These data along with the strong suggestion that protocol violations increase the chances for hemorrhage in intravenous tPA-treated patients have fostered consideration of other innovative strategies to allow patients with acute ischemic stroke access to qualified stroke expertise within the 3-hour window. Currently under investigation are "treat and ship" protocols with air transport $^{10,14}$ and telemedicine. ${ }^{40,52}$ Given the clear benefit of intravenous tPA and the relatively short time window of therapeutic opportunity, additional creative solutions will be needed.

Cost effectiveness analysis of intravenous tPA in acute ischemic stroke suggests overall savings for patient care expenditure. ${ }^{22}$ Initially, there is an increase in acute care costs by 1.7 million dollars, followed by a reduction of more than 6 million dollars in rehabilitation and nursing home care costs per 1000 eligible treated patients. In the cost analysis a savings of 564 quality-adjusted life years over 30 years was also found. Therefore, intravenous tPA when administered to treat acute ischemic stroke in appropriate patients improves functional outcome at 3 months and provides a net cost savings to the health care system.

\section{Aspirin Therapy}

Given its success in cardiovascular disease, aspirin has been examined in the acute ischemic stroke setting. In two recent large clinical trials the beneficial effect of aspirin in acute ischemic stroke has been examined., ${ }^{9,35}$ Taken together, the authors of these studies demonstrate a modest reduction of 10 deaths or recurrent strokes per 1000 patients during the first few weeks after the initial stroke. These data suggest that aspirin administered within 48 hours of an acute ischemic stroke primarily provides benefit by reducing the incidence of early recurrent stroke and not by affecting the index stroke. Therefore, aspirin is a reasonable treatment in the acute ischemic stroke setting. However, it should not be considered as an alternative to intravenous tPA in the appropriate intravenous tPA-eligible patients and cannot be administered within the first 24 hours after thrombolytic therapy.

\section{INVESTIGATIONAL AND EMERGING THERAPIES}

\section{Ancrod Therapy}

Ancrod is a purified fraction of the venom of the Malayan pit viper, which has been shown to produce rapid and effective defibrinogenation in humans. It was investigated to determine if it yielded benefit in acute ischemic stroke patients. To date, studies in which patients received Ancrod within 3 hours of symptom onset have suggested benefit. Based on the preliminary data, it appears difficult to maintain adequate plasma levels to allow for effective defibrinogenation. $^{2-4}$ The Phase III data have not been published and this agent is not FDA approved or available in the US.

\section{Neuroprotective Agents}

Recently, much interest has been focused on neuroprotective agents. Unfortunately, the results of large randomized trials in both the US and Europe have been disappointingly negative. ${ }^{18,34}$ There are numerous studies currently underway in which the efficacy of new agents is being tested.

\section{Intraarterial Thrombolytics}

Early recanalization of occluded cerebral vessels is believed to improve clinical outcomes in patients with acute ischemic strokes. Clinical experience also suggests greater recanalization rates with intraarterial thrombolysis than when using the intravenous route. ${ }^{17,50}$ Given the reported feasibility and relative safety of catheter application of thrombolytics, ${ }^{15}$ a randomized, prospective trial of an intraarterial thrombolytic was recently undertaken. The PROACT-I ${ }^{16}$ was the first randomized, double-blind, multicenter trial, in which the safety and efficacy of intraarterial delivery of recombinant pro-urokinase ( $\mathrm{r}$-proUK) was compared with placebo. The trial was conducted using a homogeneous patient population; patients presented with an acute middle cerebral artery occlusion of fewer than 6 hours duration. The PROACT-I study demonstrated that intraarterial infusion of r-proUK was associated with higher rates of arterial recanalization compared with placebo infusion.

The follow-up PROACT-II ${ }^{24}$ study was a multicenter, randomized trial in which 180 patients of 12,323 screened patients were randomized. The study patients were randomized in a ratio of 2:1 (treatment:control), and the study was not placebo controlled because of concerns regarding the infusion of a placebo drug into an occluded middle cerebral artery. In addition, the dose of r-proUK was increased from 6 to $9 \mathrm{mg}$ and the trial was changed to an open design with blinded follow-up study. From the primary efficacy data analysis, $40 \%$ of r-proUK patients compared with $25 \%$ of control patients had a modified Rankin score of 2 or less at 90 days after stroke onset ( $\mathrm{p}=$ $0.04)$. The rate of symptomatic ICH was $10 \%$ in the treatment group compared with $2 \%$ in the control group, although this did not reach statistical significance at 10 days. The increased number of ICHs did not change the mortality rates between the two groups. Despite the lack of statistical significance of the hemorrhage rate, the clin- 
ical significance is considerable. Despite the lack of FDA approval for this treatment, numerous academic centers, $,, 6,21,27,32,36,45,54,60,62$ and even small community hospitals, ${ }^{20}$ are treating patients with intraarterial thrombolysis. Direct comparison between these uncontrolled studies is difficult given that different dosing regimens, stroke types, and time intervals were examined.

A major concern regarding the use of intraarterial thrombolysis is the amount of time needed to obtain an emergency cerebral angiogram. Intravenous tPA may be given with minimal delay once a head CT is obtained, but many patients are still left with residual neurological deficits. ${ }^{48}$ In addition, many patients treated with intravenous tPA have residual occlusion of the involved vessel. ${ }^{8}$ An innovative treatment approach in which an attempt was made to combine initial intravenous tPA with local intraarterial thrombolysis was used in the Emergency Management of Stroke Bridging Trial. ${ }^{4}$ This was a double-blind, randomized, placebo-controlled, multicenter feasibility study with 35 enrolled patients, in which intravenous plus intraarterial tPA (IV/IA) treatment was compared with placebo plus intraarterial tPA (placebo/IA). The study was conducted prior to the FDA approval of intravenous t-PA. Despite randomization, patients who were found not to have an arterial thrombus at the time of the angiogram did not receive intraarterial tPA treatment. The rate of symptomatic ICH at 72 hours ranged from $5.5 \%$ (IV/IA Group) to $11.8 \%$ (placebo/IA Group). Primary outcome measures were not different between treatment groups at 90 days of follow-up study. This study showed a direct correlation between the National Institutes of Health stroke scale score and the presence of thrombus in a "major cerebral artery." 41 This finding could be explored in future studies as a marker to identify patients who may benefit from intraarterial thrombolysis based on the PROACT data. ${ }^{24}$ Because the number of patients was so small in the Emergency Management of Stroke Bridging Trial, no definitive conclusions can be drawn except that combination intravenous/intraarterial therapy is feasible and appears safe and deserves further investigation. Authors of other recent publications have proposed the combined use of intraarterial thrombolysis and subsequent endovascular interventions such as intracranial angioplasty. ${ }^{13,57,58}$ These preliminary reports are interesting but need further exploration in rigorous clinical trials.

\section{Combined Carotid Endarterectomy and Intraarterial Thrombolysis}

To help reduce operative risks incurred by patients undergoing CEA, proper patient selection is made according to published guidelines. Even with proper patient selection, perioperative strokes still occur in up to $2 \%$ of patients. ${ }^{33}$ When a postoperative stroke does occur, however, the patient is ineligible for intravenous thrombolysis according to current NINDS guidelines. ${ }^{48}$ Authors of a recent small case series reported on five CEA patients who underwent intraarterial thrombolysis after being reexamined for development of an acute ischemic stroke in the immediate post-operative period. Four of five patients had "negligible deficits" at the time of hospital discharge and no ICHs were identified. ${ }^{61}$ Further studies on this and other postoperative patient populations are necessary. ${ }^{38}$

\section{Emergency CEA Surgery}

Another area of growing interest in the CEA population is emergency CEA surgery for acute ischemic stroke treatment. The effects and benefits of CEA surgery in patients with severe carotid stenosis have been demonstrated;44,49 whether patients with acute symptomatic carotid occlusion would benefit from surgery is unclear. Emergency CEA surgery is considered high risk because of earlier reports describing poor outcomes and operative mortality rates approaching $50 \% .{ }^{26}$ Several recent uncontrolled case series of emergency CEA procedures, however, suggest that the morbidity and mortality rates may not be as high as previously reported, possibly because of better patient selection. ${ }^{19,43,51}$

\section{CONCLUSIONS}

Acute ischemic stroke must be considered a medical emergency that requires immediate and accurate diagnosis and rapid and appropriate management. Recent welldesigned and executed clinical trials have definitively demonstrated that in the appropriate patient population intravenous tPA administered within 3 hours of symptom onset provides significant functional improvement and decreases health care system costs. It is important to point out that although the ICH rate in patients administered intravenous tPA is 10-fold higher than in control patients, mortality rates do not increase and, overall, all previously studied patient populations who received intravenous tPA fared better than those who did not receive intravenous t-PA. However, deviations from the NINDS protocol for administration of intravenous tPA in acute ischemic stroke do dramatically increase the chance of ICH. Therefore, time is of the essence in those patients with ischemic stroke who present for care within 3 hours of symptom onset; they must be rapidly considered for intravenous tPA. Intravenous tPA is the only FDA approved therapy at this time for acute ischemic stroke.

The care for patients who present after the 3-hour window or for patients who are otherwise ineligible for intravenous tPA is more controversial and nebulous. The results of the recent PROACT II trial do suggest clinical benefit of intraarterial r-proUK in middle cerebral artery distribution ischemic strokes within 6 hours of symptom onset. However, the lack of FDA approval of r-proUK, the current lack of availability of the drug, and differences in institutional neurointerventional radiology expertise have limited the use of intraarterial therapy. Other neurointerventional techniques are intriguing but anecdotal and require further well-designed prospective trials to assess feasibility, safety, and efficacy.

\section{Note Added in Proof}

The Ancrod data was published in JAMA on May 10, 2000 (Sheldon, DG et al: JAMA 283:239-2403, 2000).

\section{Appendix}

NINDS Trial and Treatment Protocols ${ }^{2}$

Patients Included.

1) Symptoms consistent with an ischemic stroke and a clearly defined time of onset of 3 hours or less. 
2) A deficit measurable on the National Institutes of Health scale score.

3) A baseline CT scan of the brain showing no evidence of intracranial hemorrhage.

Patients Excluded.

1) Prior stroke or serious head trauma within the preceding 3 months.

2) Major surgery within 14 days

3) History of intracranial hemorrhage.

4) Systolic blood pressure above $185 \mathrm{~mm} \mathrm{Hg}$ or diastolic

blood pressure above $110 \mathrm{~mm} \mathrm{Hg}$.

5) Rapidly improving or minimal symptoms.

6) Symptoms suggestive of subarachnoid hemorrhage.

7) Gastrointestinal hemorrhage or urinary tract hemorrhage within the previous 21 days.

8) Arterial puncture at a noncompressible site within the previous 7 days.

9) Seizure at the onset of stroke.

10) Taking anticoagulants or received heparin within the 48 hours preceding the onset of stroke and elevated partial thromboplastin times greater than 15 seconds.

11) Platelet counts below $100,000 \mathrm{p} / \mathrm{ml}^{3}$.

12) Glucose concentrations below $50 \mathrm{mg} \mathrm{p} / \mathrm{dl}(2.7 \mathrm{mmol} \mathrm{p} / \mathrm{L})$ or above $400 \mathrm{mg} \mathrm{p} / \mathrm{dl}(22.2 \mathrm{mmol} \mathrm{p} / \mathrm{L})$

13) Aggressive treatment required to maintain blood pressure within the specified limits.

Treatment.

1) Intravenous tPA, $0.9 \mathrm{mg} \mathrm{p} / \mathrm{kg}$ of body weight (maximum $90 \mathrm{mg}$ ), $10 \%$ administered as a bolus followed by delivery of the remaining $90 \%$ as a constant infusion over a period of 60 minutes.

2) No anticoagulants or antiplatelet agents given for 24 hours after treatment.

3) Systolic blood pressure carefully maintained below 185 $\mathrm{mm} \mathrm{Hg}$ and diastolic blood pressure below $110 \mathrm{~mm} \mathrm{Hg}$ for 24 hours after treatment but avoiding hypotension.

4) Monitoring for 24 hours posttreatment in an intensive care unit with frequent neurological examinations.

\section{Disclosure}

Dr. Johnston is a paid consultant and speaker for Genentech Inc., South San Francisco, CA.

\section{Acknowledgment}

Dr. Worrall is supported in part by a grant from the American Academy of Neurology Clinical Research Training Fellowship. Drs. Kindler and Lopez contributed equally to this manuscript.

\section{References}

1. Albers GW, Bates VE, Clark WM, et al: Intravenous tissue-type plasminogen activator for treatment of acute stroke: the Standard Treatment with Alteplase to Reverse Stroke (STARS) study. JAMA 283: 1145-1150, 2000

2. The Ancrod Stroke Study Investigators: Ancrod for the treatment of acute ischemic brain infarction. Stroke 25:1755-1759, 1994

3. Atkinson RP: Ancrod in the treatment of acute ischemic stroke. Drugs 54 (Suppl 3): 100-108, 1994

4. Atkinson RP: Ancrod in the treatment of acute ischemic stroke. A review of clinical data. Cerebrovasc Dis 8 (Suppl 1):23-28, 1998

5. Bendszus M, Urbach H, Ries F, et al: Outcome after local intraarterial fibrinolysis compared with the natural course of patients with a dense middle cerebral artery on early CT. Neuroradiology 40:54-58, 1998

6. Brandt T, von Kummer R, Muller-Kuppers M, et al: Thrombolytic therapy of acute basilar artery occlusion. Variables affecting recanalization and outcome. Stroke 27:875-881, 1996
7. Broderick J, Brott T, Kothari R,, et al: The Greater Cincinnati/ Northern Kentucky Stroke Study: preliminary first-ever and total incidence rates of stroke among blacks. Stroke 29:415-321, 1998

8. Brott TG, Haley EC Jr, Levy DE, et al: Urgent therapy for stroke. Part I. Pilot study of tissue plasminogen activator administered within 90 minutes. Stroke 23:632-640, 1992

9. CAST (Chinese Acute Stroke Trial) Collaborative Group: CAST: randomised placebo-controlled trial of early aspirin use in 20,000 patients with acute ischaemic stroke. Lancet 349: 1641-1649, 1997

10. Chalela JA, Kasner SE, Jauch EC, et al: Safety of air medical transportation after tissue plasminogen activator administration in acute ischemic stroke. Stroke 30:2366-2368, 1999

11. Chiu D, Krieger D, Villar-Cordova C, et al: Intravenous tissue plasminogen activator for acute ischemic stroke: feasibility, safety, and efficacy in the first year of clinical practice. Stroke 29:18-22, 1998

12. Clark WM, Wissman S, Albers GW, et al: Recombinant tissuetype plasminogen activator (Alteplase) for ischemic stroke 3 to 5 hours after symptom onset. The ATLANTIS Study: a randomized controlled trial. JAMA 282:2019-2026, 1999

13. Connors JJ III, Wojak JC: Percutaneous transluminal angioplasty for intracranial atherosclerotic lesions: evolution of technique and short-term results. J Neurosurg 91:415-423, 1999

14. Conroy MB, Rodriguez SU, Kimmel SE, et al: Helicopter transfer offers a potential benefit to patients with acute stroke. Stroke 30:2580-2584, 1999

15. del Zoppo GJ, Ferbert A, Otis S, et al: Local intra-arterial fibrinolytic therapy in acute carotid territory stroke. A pilot study. Stroke 19:307-313, 1988

16. del Zoppo GJ, Higashida RT, Furlan AJ, et al: PROACT: a phase II randomized trial of recombinant pro-urokinase by direct arterial delivery in acute middle cerebral artery stroke. PROACT Investigators. Prolyse in Acute Cerebral Thromboembolism. Stroke 29:4-11, 1998

17. del Zoppo GJ, Pessin MS, Mori E, et al: Thrombolytic intervention in acute thrombotic and embolic stroke. Semin Neurol 11:368-384, 1991

18. Devuyst G, Bogousslavsky J: Clinical trial update: neuroprotection against acute ischaemic stroke. Curr Opin Neurol 12: 73-79, 1999

19. Eckstein HH, Schumacher H, Klemm K, et al: Emergency carotid endarterectomy. Cerebrovasc Dis 9:270-281, 1999

20. Edwards MT, Murphy MM, Geraghty JJ, et al: Intra-arterial cerebral thrombolysis for acute ischemic stroke in a community hospital. AJNR 20:1682-1687, 1999

21. Endo S, Kuwayama N, Hirashima Y, et al: Results of urgent thrombolysis in patients with major stroke and atherothrombotic occlusion of the cervical internal carotid artery. AJNR 19: 1169-1175, 1998

22. Fagan SC, Morgenstern LB, Petitta A, et al: Cost-effectiveness of tissue plasminogen activator for acute ischemic stroke. NINDS rt-PA Stroke Study Group. Neurology 50:883-890, 1998

23. Ford G, Freemantle N: ECASS-II: intravenous alteplase in acute ischaemic stroke. European Co-operative Acute Stroke Study-II. Lancet 353:65-68 1999

24. Furlan A, Higashida R, Wechsler L, et al: Intra-arterial prourokinase for acute ischemic stroke. The PROACT II study: a randomized controlled trial. JAMA 282:2003-2011, 1999

25. Goldstein LB, Hey LA, Laney R: North Carolina stroke prevention and treatment facilities survey: rtPA therapy for acute stroke. Stroke 29:2069-2072, 1998

26. Goldstone J, Moore WS: Emergency carotid artery surgery in neurologically unstable patients. Arch Surg 111:1284-1291, 1976

27. Gonner F, Remonda L, Mattle H, et al: Local intra-arterial 
thrombolysis in acute ischemic stroke. Stroke 29:1894-1900, 1998

28. Grond M, Rudolf J, Schmulling S, et al: Early intravenous thrombolysis with recombinant tissue-type plasminogen activator in vertebrobasilar ischemic stroke. Arch Neurol 55: 466-469, 1998

29. Grond M, Stenzel C, Schmulling S, et al: Early intravenous thrombolysis for acute ischemic stroke in a community-based approach. Stroke 29:1544-1549, 1998

30. Hacke W, Brott T, Caplan L, et al: Thrombolysis in acute ischemic stroke: controlled trials and clinical experience. Neurology 53 (7 Suppl 4):S3-S14, 1999

31. Hacke W, Kaste M, Fieschi C, et al: Randomised double-blind placebo-controlled trial of thrombolytic therapy with intravenous alteplase in acute ischaemic stroke (ECASS II). Second European-Australasian Acute Stroke Study Investigators. Lancet 352:1245-1251, 1998

32. Hacke W, Zeumer H, Ferbert A, et al: Intra-arterial thrombolytic therapy improves outcome in patients with acute vertebrobasilar occlusive disease. Stroke 19:1216-1222, 1988

33. Hertzer NR, O'Hara PJ, Mascha EJ, et al: Early outcome assessment for 2228 consecutive carotid endarterectomy procedures: the Cleveland Clinic experience from 1989 to 1995. J Vasc Surg 26:1-10, 1997

34. Hickenbottom SL, Grotta J: Neuroprotective therapy. Semin Neurol 18:485-492, 1998

35. The International Stroke Trial Collaborative Group: The International Stroke Trial (IST): a randomised trial of aspirin, subcutaneous heparin, both, or neither among 19435 patients with acute ischaemic stroke. Lancet 349:1569-1581, 1997

36. Jahan R, Duckwiler GR, Kidwell CS, et al: Intraarterial thrombolysis for treatment of acute stroke: experience in 26 patients with long-term follow-up. AJNR 20:1291-1299, 1999

37. Katzan IL, Furlan AJ, Lloyd LE, et al: Use of intravenous tissue-type plasminogen activator for acute stroke: the Cleveland area experience. JAMA 283:1151-1158, 2000

38. Katzan IL, Masaryk TJ, Furlan AJ, et al: Intra-arterial thrombolysis for perioperative stroke after open heart surgery. Neurology 42:1081-1084, 1999

39. Kwiatkowski TG, Libman RB, Frankel M, et al: Effects of tissue plasminogen activator for acute ischemic stroke at one year. National Institute of Neurological Disorders and Stroke Recombinant Tissue Plasminogen Activator Stroke Study Group. N Engl J Med 340:1781-1787, 1999

40. Levine SR, Gorman M: "Telestroke": the application of telemedicine for stroke. Stroke 30:464-469, 1999

41. Lewandowski CA, Frankel M, Tomsick TA, et al: Combined intravenous and intra-arterial r-TPA versus intra-arterial therapy of acute ischemic stroke: Emergency Management of Stroke (EMS) Bridging Trial. Stroke 30:2598-2605, 1999

42. Marler JR, Tilley BC, Lu M, et al: The NINDS rt-PA Stroke Study Group. Earlier treatment associated with better outcome in the NINDS tPA Stroke Study. Stroke 30:244, 1999 (Abstract)

43. Mead GE, O'Neill PA, McCollum CN: Is there a role for carotid surgery in acute stroke? Eur J Vasc Endovasc Surg 13: $112-121,1997$

44. Moore WS, Barnett HJ, Beebe HG, et al: Guidelines for carotid endarterectomy. A multidisciplinary consensus statement from the ad hoc Committee, American Heart Association. Stroke 26:188-201, 1995

45. Mori E, Tabuchi M, Yoshida T, et al: Intracarotid urokinase with thromboembolic occlusion of the middle cerebral artery. Stroke 19:802-812, 1988

46. National Institutes of Neurological Disorders and Stroke rt-PA Stroke Study Group: Generalized efficacy of t-PA for acute stroke. Subgroup analysis of the NINDS t-PA Stroke Trial. Stroke 28:2119-2125, 1997
47. National Institutes of Neurological Disorders and Stroke rt-PA Stroke Study Group: Intracerebral hemorrhage after intravenous t-PA therapy for ischemic stroke. Stroke 28:2109-2118, 1997

48. National Institutes of Neurological Disorders and Stroke rt-PA Stroke Study Group: Tissue plasminogen activator for acute ischemic stroke. The National Institute of Neurological Disorders and Stroke rt-PA Stroke Study Group. N Engl J Med 333:1581-1587, 1995

49. North American Symptomatic Carotid Endarterectomy Trial Collaborators: Beneficial effect of carotid endarterectomy in symptomatic patients with high-grade carotid stenosis. N Engl J Med 325:445-453, 1991

50. Sasaki O, Takeuchi S, Koike T, et al: Fibrinolytic therapy for acute embolic stroke: intravenous, intracarotid, and intra-arterial local approaches. Neurosurgery 36:246-53, 1995

51. Schneider C, Johansen K, Konigstein R, et al: Emergency carotid thromboendarterectomy: safe and effective. World J Surg 23:1163-1167, 1999

52. Shafqat S, Kvedar JC, Guanci MM, et al: Role for telemedicine in acute stroke. Feasibility and reliability of remote administration of the NIH stroke scale. Stroke 30:2141-2145, 1999

53. Steiner T, Bluhmki E, Kaste M, et al: The ECASS 3-hour cohort. Secondary analysis of ECASS data by time stratification. ECASS Study Group. European Cooperative Acute Stroke Study. Cerebrovasc Dis 8:198-203, 1998

54. Suarez JI, Sunshine JL, Tarr R, et al: Predictors of clinical improvement, angiographic recanalization, and intracranial hemorrhage after intra-arterial thrombolysis for acute ischemic stroke. Stroke 30:2094-2100, 1999

55. Tanne D, Bates VE, Verro P, et al: Initial clinical experience with intravenous tissue plasminogen activator for acute ischemic stroke: a multicenter survey. The t-PA Stroke Survey Group. Neurology 53:424-427, 1999

56. Tanne D, Gorman MJ, Bates VE, et al: Intravenous tissue plasminogen activator for acute ischemic stroke in patients aged 80 years and older: the tPA stroke survey experience. Stroke 31: $370-375,2000$

57. Ueda T, Hatakeyama T, Kohno K, et al: Endovascular treatment for acute thrombotic occlusion of the middle cerebral artery: local intra-arterial thrombolysis combined with percutaneous transluminal angioplasty. Neuroradiology 39:99-104, 1997

58. Ueda T, Sakaki S, Nochide I, et al: Angioplasty after intra-arterial thrombolysis for acute occlusion of intracranial arteries. Stroke 29:2568-2574, 1998

59. Wang DZ, Rose JA, Honings DS, et al: Treating acute stroke patients with intravenous tPA. The OSF stroke network experience. Stroke 31:77-81, 2000

60. Wijdicks EF, Nichols DA, Thielen KR, et al: Intra-arterial thrombolysis in acute basilar artery thromboembolism: the initial Mayo Clinic experience. Mayo Clin Proc 72:1005-1013, 1997

61. Winkelaar GB, Salvian AJ, Fry PD, et al: Intraoperative intraarterial urokinase in early postoperative stroke following carotic endarterectomy: a useful adjunct. Ann Vasc Surg 13:566-570, 1999

62. Zeumer H, Freitag HJ, Zanella F, et al: Local intra-arterial fibrinolytic therapy in patients with stroke: urokinase versus recombinant tissue plasminogen activator (r-TPA). Neuroradiology 35:159-162, 1993

Manuscript received March 20, 2000.

Accepted in final form May 1, 2000.

Address reprint requests to: Karen C. Johnston, M.D., M.Sc., University of Virginia Health System, Department of Neurology \#800394, Charlottesville, Virginia 22908. email: kj4v@virginia .edu. 